sung, »dass eine Abschaffung der Gesetzgebungskompetenz des Bundes im Bereich Strafvollzug verfehlt wäre." Der Abgeordnete Dr. Klaus Lederer hält die Abschaffung der Bundeskompetenz auch unter dem Gesichtspunkt der Gleichbehand- lung von Strafgefangenen, die nach gleichen Strafgesetzen bestraft werden für äußerst problematisch, hält jedoch die Möglichkeiten seiner eigenen Einflussnahme für gering, zumal die PDS bei der Besetzung der Föderalismuskommission nicht berücksichtigt worden war.

Man wird darüber streiten können, ob diese Reaktionen eher ermutigend oder ernüchternd sind. Der Aufruf hat allemal seinen Zweck er- füllt über Konsequenzen und mögliche Gefahren zu informieren, einen Rechtfertigungsdruck zu erzeugen und das Thema Strafvollzug nicht als gar zu leichten Spielball zu benutzen.

\section{Kriminologie zwischen Kritik und Kooperation}

\section{Große Resonanz auf Kriminologietag in Frankfurt (Main)}

Michael Jasch

$\mathrm{D}$ er rechtspolitische Zug scheint unaufhaltsam auf einem Kurs von Punitivität und Kontrolle zu sein. Kriminalpolitiker beschwören die »Sicherheit« wie eine Ikone. Ob die Ausweitung der Sicherungsverwahrung, mehr und längere Haftstrafen, DNS-Karteien oder der staatliche Zugriff auf die Kontodaten jedes Bürgers - alles scheint im Dienste des eher affektiven als präzise bestimmbaren Begriffes der Sicherheit willkommen zu sein. An kriminologischempirischen Belegen für ihre Forderungen und Initiativen sind die kriminalpolitischen Akteure meist ebenso wenig interessiert wie die breite Öffentlichkeit. Die Sorge von Bürgern über Kriminalität hält sich trotz konstanter oder rückläufiger Kriminalität auf hohem Niveau. Eine breite öffentliche Opposition gegen die stetigen Ausweitungen von polizeilichen Eingriffsbefugnissen und Datensammlungen sucht man im deutschsprachigen Raum vergebens.

Angesichts dieser gesellschaftlichen Großwetterlage war es überfällig, dass die Kriminologen der großen Fachorganisationen im deutschsprachigen Raum gemeinsam ihr Verhältnis zu Prävention und Sicherheit sowie die Situation ihrer Wissenschaft in der Ausbildung sämtlicher im Bereich des Kriminaljustizsystems tätigen Berufsgruppen diskutierten. Diese beiden Themen bildeten die Schwerpunkte des ersten Kriminologietages, zu dem 130 Wissen- schaftler und Justizpraktiker aus den Reihen der Gesellschaft für interdisziplinäre wissenschaftliche Kriminologie (GiwK), der Neuen Kriminologischen Gesellschaft (NKG) und der Schweizerischen Arbeitsgruppe für Kriminologie (SAK) in Frankfurt am Main zusammen gekommen sind. Die Sorge um einen »drohenden oder schon vollzogenen Bedeutungsverlust « der Kriminologie, wie es im Tagungsaufruf hieß, hatte acht Hochschullehrer und -lehrerinnen zu dieser Initiative bewogen. Die hinter den skizzierten Themen liegenden Fragen sind wissenschaftstheoretisch keineswegs neu, aber hochgradig aktuell: Durch wen oder was wird der Gegenstand der Kriminologie - sei es in den Lehrbüchern, der Forschung oder den Fachdiskursen heute eigentlich bestimmt? Ist die wissenschaftliche Distanz der Kriminologen noch ausreichend gesichert, wenn sich die Profession zu einem großen Teil mit Auftragsund Begleitforschung zu aktuellen staatlichen Projekten verdingt und dabei mehr oder weniger reflektiert sowohl die Gegenstände als auch die Terminologie dieser Initiativen übernimmt? An den in jüngerer Zeit zunehmenden Thematisierungen von "gemeingefährlichen Tätern « (so das Titelthema von Heft 4 der Neuen Kriminalpoltik 2004), sogenannten Intensivtätern oder des Islamismus als einer innerstaatlichen Bedrohung (vgl. Bundesministerium des Inneren: Texte zur Inneren Sicherheit 2003) lässt sich ablesen, wie nah der kriminologi- sche Diskurs zumindest an die Rhetorik des kriminalpolitischen Zeitgeistes heran gekommen ist. Natürlich entscheidet sich erst mit der Vorgehensweise und Ausrichtung dieser Arbeiten, ob und in welchem Umfang der Kriminologe als "Bedarfsforscher" (vgl.: M. Walter, in: NeUe KRIMINALPOLITIK 2000, Heft 1) agiert - und wenn ja, dann für wessen Bedarf.

In Frankfurt spitzte ein mit Martin Killias, Friedrich Lösel, Cornelius Prittwitz und Fritz Sack besetztes Podium die Frage - etwas zu schnell auf das Verhältnis zwischen Empirie und Theorie zu. So sprach sich Friedrich Lösel (Erlangen-Nürnberg) für eine (noch) stärkere Bedeutung empirischer Forschung aus: "Wissenschaft muss vor allem empirisch arbeiten", meinte er. Dies gelte auch für den Bereich der Kriminalprävention, die eine Form des gesellschaftlichen Risikomanagements darstelle, bei deren Evaluation stets ethische, rechtliche und politische Zusammenhänge "mitgedacht « und damit auch immanent bewertet werden müssten. Dass speziell die »deutsche Kriminologie überaus lehrbuchlastig « sei, bemängelte Martin Killias (Lausanne), der sich ebenfalls für eine Verstärkung der empirischen Forschungsanstrengungen stark machte.

Dass dennoch nicht ohne theoretische Fundierung auszukommen ist, machte Fritz Sack deutlich, der davor warnte, »die Empirie durch das Nadelöhr der Theorie zu zwängen «. Angesichts der sich radikal wandelnden europäischen Gesellschaften sieht Sack die künftigen Aufgaben der Kriminologie unter anderem in einer stärkeren Befassung mit neuen Gegenstandsbereichen: Nicht vornehmlich die traditionell im Fokus stehenden Gruppen von Tätern und Opfern, sondern die Politik, die privaten
Sicherheitsindustrien und die Rolle der Medien sollten stärker als bislang in das Blickfeld der Kriminologie gelangen. Diesen Punkt griff später Karl F. Schumann auf, der die Einrichtung eines gemeinsamen »Arbeitskreises Medien« als Schnittstelle zwischen kriminologischer Forschung und Massenmedien anregte, und damit auf große Zustimmung stieß. Für eine Überwindung der überkommenen Gräben zwischen kritischen und praxisorientierten Kriminologen sowie den unterschiedlich ausgerichteten Fachgesellschaften sprach sich Cornelius Prittwitz (Frankfurt a.M.) mit Nachdruck aus. Kriminologie müsse sich heute sowohl mit den Ursachen und der Prävention von Kriminalität befassen als auch dem Anspruch der Wissenschaftlichkeit folgend - stets kritisch sein. Gerade das gegenwärtig zu konstatierende Desinteresse der Kriminalpolitiker könne, vielleicht paradoxerweise, eine größere Einigkeit und Kooperation zwischen den an ihrer gesellschaftlichen Wahrnehmung interessierten Kriminologen bewirken.

Gegen ein »verbreitetes Jammern « unter den Kriminologen über die eigene Wirkungslosigkeit wandte sich Christian Pfeiffer (Hannover), der stattdessen von seinen Kolleginnen und Kollegen deutlichere Schritte in die Öffentlichkeit und ein offensiveres Zugehen auf die kriminalpolitisch Verantwortlichen forderte. Auf diesen Wegen gebe es durchaus, wie Pfeiffer anhand von Beispielen illustrierte, reale Chancen, kriminologische Erkenntnisse wirksam werden zu lassen. Dieser Einschätzung schloss sich Kai-D. Bussmann (Halle) an, der zudem auf einen Mangel engagierter und qualifizierter Kriminologen an den Lehrstühlen hinwies: »Es gibt viel zu wenige Leute an den Hochschulen, die Kriminologie betreiben und nicht nur lesen wol- 
len«. Auch Rüdiger Lautmann (Hamburg) warnte, es sei eine Illusion zu glauben, die Empirie von der kriminalpolitischen Agenda entkoppeln zu können: »Empirie, die am Meinungsklima vorbeigeht, wird nicht gehört «, sagte Lautmann. Dagegen mahnte Klaus Boers (Münster) bei aller tagesaktuellen Ausrichtung die Notwendigkeit einer grundlagenorientierten und theoriegeleiteten Forschung an. Ausgangspunkt jeder Forschung müsse die Untersuchung grundlegender Zusammenhänge sein. Zugleich betonte er, dass es nicht weiterführen könne, auch nur implizit von einem vermeintlichen Gegensatz zwischen Theorie und Empirie auszugehen. Nur, wenn diese Begriffe weder in ein Gegensatz- noch in ein Subordinationsverhältnis gestellt werden, könne kriminologische Forschung zu echten Erkenntnisgewinnen führen.

Wie der Wissenstransfer in die Praxis aussehen kann und welche Probleme damit verbunden sind, diskutierte ein zweites Podium mit Hans-Jürgen Kerner, Karl F. Schumann, Christian Pfeiffer und Arno Pilgram anhand des Periodischen Sicherheitsberichtes (PSB). Derzeit beginnen in Deutschland die Arbeiten an einer zweiten Version dieses 2001 erstmals erschienenen Berichtes der Bundesregierung, an dem fünf Hochschullehrer für Kriminologie maßgeblich mitgewirkt hatten. Dabei äußerte Kerner, er hielte ein gänzlich unabhängiges Gremium als Produzenten derartiger Lageberichte für eine vorzugswürdige Lösung. Während der Diskussion mit dem Plenum stellte sich schnell heraus, dass es sich bei dem PSB vornehmlich um ein universitäres Thema handelt. Sowohl Praktiker als auch Fachhochschuldozenten berichteten, die Ergebnisse dieses Projektes würden in ihren Institutionen so gut wie überhaupt nicht rezipiert.

Bevor Hans-Jürgen Kerner mit einer umfassenden Bestandsaufnahme der aktuellen Situation der Kriminologie und einer Bilanz des Kriminologietages die Veranstaltung abschloss, stand die Situation der Kriminologie in der Ausbildung im Mittelpunkt der Tagung. Sowohl den Ist-Zustand als auch die Perspektiven einer Verankerung kriminologischer Themen in der Aus- bildung künftiger Juristen und Polizisten, Sozialwissenschaftler und Sozialarbeiter, Psychiater und Psychologen diskutierten die Teilnehmer in gesonderten Arbeitskreisen. Der in Deutschland ganz überwiegenden Verortung der Kriminologie an den juristischen Fakultäten entsprechend, stießen die Diskussionen über den Standort der Kriminologie in diesem Bereich der Ausbildung auf das Interesse der meisten Wissenschaftler. Gegenstand der Diskussionen unter Leitung von Jörg-Martin Jehle waren vor allem die aktuellen Perspektiven, die sich aus den in den Bundesländern geschaffenen Möglichkeiten für ein von den Universitäten eigenständig organisiertes Schwerpunktstudium ergeben.

Ein eher düsteres Bild der Lage zeichneten die Teilnehmer der Arbeitsgruppe, die sich unter Leitung von Dieter Hermann (Heidelberg) mit der Relevanz der Kriminologie in den sozialwissenschaftlichen Studiengängen befasste. Sowohl in der universitären Lehre als auch den soziologischen Fachjournalen und -verbänden spiele diese Disziplin eine höchst untergeordnete Rolle. Die Gründe dafür wurden einerseits in der spezifischen Ausrichtung der deutschen Sozialwissenschaften, andererseits aber auch in der Kriminologie selbst, die neuere soziologische Entwicklungen kaum rezipieren und eigene Ergebnisse selten in die aktuellen Diskussionsprozesse der Soziologen einbringe, gesehen. Hermann regte neben der Gründung kriminalsoziologischer Sektionen in den Fachverbänden auch an, die Universitäten mögen ihr Lehrangebot für die Studierenden der unterschiedlichen Fachrichtungen stärker interdisziplinär öffnen und die Studienleistungen gegenseitig anerkennen.

Als verbesserungsbedürftig sahen auch die Lehrenden an Fachhochschulen für Sozialpädagogik und soziale Arbeit die Stellung der Kriminologie an ihren Einrichtungen an. Derzeit, so konstatierten die Teilnehmer dieses Arbeitskreises unter der Diskussionsleitung von Gabriele Kawamura-Reindl (Nürnberg), sei kein einziges Fachhochschul-Curriculum bekannt, in dem die Kriminologie als eigenes Fach explizit vorhanden ist. Wenn überhaupt, würden Frage- stellungen aus dieser Wissenschaft nur im Rahmen allgemeiner Einführungen in juristische und soziologische Grundlagen oder von individuellen Studienschwerpunkten der Studierenden angesprochen. Dabei böten gerade jetzt die aus dem europäischen Bologna-Prozess hervorgegangenen neuen Studiengänge an den Fachhochschulen die Chance, die Kriminologie als interdisziplinäres Fach stärker als bisher in die Bachelor- oder Masterstudiengänge zu integrieren. Unabdingbar sei jedoch eine Verbesserung der materiellen und personellen Ausstattung an den Einrichtungen, die derzeit kaum eigene Forschungsarbeiten zulasse. Für künftige Kriminologietage und ähnliche Fachtagungen wünschten sich die Teilnehmer die Einrichtung einer Arbeitsgruppe, mit der die Kommunikation zwischen den Lehrkräften an den Fachhochschulen gefördert wird.

Ganz anders ist gegenwärtig die Kriminologie im Rahmen der Ausbildung von Polizeibeamten positioniert, wie eine von BKA-Vizepräsident Jürgen Stock moderierte Gruppe herausfand. In den Ausbildungsgängen für den höheren und gehobenen Kriminaldienst gewinne die Kriminologie derzeit sogar an Bedeutung. Im Bundeskriminalamt etwa wurde diese Wissenschaft kürzlich zu Lasten der juristischen Inhalte ausgebaut, berichtete Jürgen Stock. Auch im Vergleich zur Soziologie, der noch immer das negative Image eines polizeikritischen Faches anhafte, stehe die Kriminologie gut da. Trotz einer angespannten Haushaltslage sei auch auf Landesebene eine Erweiterung der kriminalistisch-kriminologischen Forschungsstellen $\mathrm{zu}$ verzeichnen. Jedoch werde in den Ausbildungseinrichtungen ein Spannungsverhältnis zwischen Theorie und Praxis diskutiert: Anzutreffen sei die Sorge, dass die Kriminologie zu wenig »Handwerkszeug « für die Beamten vermittele oder durch ihr wissenschaftliches Hinterfragen sogar zu einer Handlungsunsicherheit bei den Polizisten führe. Die Teilnehmer dieses Arbeitskreises erwarteten von der Wissenschaft überwiegend »Zahlen, Fakten und Lösungen im Sinne von Handlungsorientierungen, nicht aber eine moralisierende oder ideologisierende Kriminologie «, berichtete Stock.
Während des Kriminologietages haben sich drei Punkte heraus kristallisiert, die sowohl die gesellschaftspolitische Stellung dieser Disziplin als auch die Diskussionskultur ihrer Repräsentanten berühren: - An einem regelmäßigen, institutionalisierten Forum der gesamten deutschsprachigen Kriminologie, über die Grenzen der in ihren Schwerpunkten unterschiedlich ausgerichteten Fachgesellschaften hinweg, besteht aktuell zugleich ein kaum übersehbarer Bedarf und ein erhebliches Interesse unter den Kriminologen. In den beteiligten Vereinigungen scheint einer Mehrheit der Mitglieder die unmittelbaren Diskussionen innerhalb der eigenen Organisation allein nicht mehr auszureichen, auch wenn die Fachgesellschaften aufgrund ihrer unterschiedlichen Orientierungen weiterhin ihre Existenzberechtigung haben. Doch die Grenzen zwischen den Anhängern einer »kritischen Kriminologie « und den Ätiologen lassen sich oft nicht mehr so eindeutig und fraktionsartig ausmachen, wie dies vor einigen Jahrzehnten noch der Fall war. Das Feld zwischen den denkbaren Polen von pragmatischer Politikberatung und radikalkonstruktivistischer Kritik ist bunter geworden. Den Akteuren gemein ist ihre zunehmende Sorge um die Abkopplung des kriminalpolitischen Alltagshandelns von den wissenschaftlichen Einsichten.

- Um einer solchen Abkopplung entgegenzuwirken ist es vor allem erforderlich, sich den Herausforderungen zu stellen, die eine massenmedial vermittelte, geprägte und zum Teil sogar generierte Kriminalpolitik an Wissenschaft und Lehre stellt. Die Frage, wie eine auf Rationalität, empirischen Belegen und rechtsstaatlicher Freiheit basierende Kriminalpolitik, die zugleich überzeugende Antworten auf stabile emotionale Strömungen und Bedürfnisse in der breiten Bevölkerung bereithält aussehen kann, wird die Kriminologie in den nächsten Jahren vordringlich beschäftigen. Hier haben die Wissenschaftler eine Bringschuld $\mathrm{zu}$ begleichen. Ebenso dringlich stellt sich den Kriminologen allerdings die grundsätzlichere Aufgabe, von Politik und Öffentlichkeit 
mit ihren Erkenntnissen gehört $\mathrm{zu}$ werden, ohne ihre wissenschaftliche Distanz auf dem »Altar« der Mediengesellschaft zu opfern.

- Grundvoraussetzung einer wissenschaftlich fundierten Mitgestaltung ist eine starke Kriminologie an den Universitäten, Fachhochschulen und Ausbildungseinrichtungen möglichst aller Berufsfelder, die mit den »Kriminalität« genannten Phänomenen in Berührung kommen. Einhellig warnten verschiedene Redner während des Kriminologietages davor, die Kriminologie im Zuge von Sparoder Umstrukturierungsmaßnahmen in den Curricula oder Lehrstuhlwidmungen zurückzudrängen oder gar verschwinden $\mathrm{zu}$ lassen. Insbesondere die strafjuristische Ausbildung könne ohne ihre kriminologischen und rechtssoziologischen Bezüge nicht sinnvoll betrieben werden. Eine starke Kriminologie ist wiederum angewiesen auf qualifizierte Forscher unterschiedlicher Disziplinen, die diese Wissenschaft auch tatsächlich be- und vorantreiben. Dafür reicht es nicht aus, sich mit der drohenden Situation zufrieden zu geben, dass - wie es ein Tagungsbesucher formulierte - »jeder, der ein Kriminologiebuch vorlesen kann, auch Kriminologie macht, nur weil es eben zufällig auf dem Lehrplan steht«. Durch die engagiert geführten Diskussionen und die deutliche Annäherung zwischen Mitgliedern der großen Fachgesellschaften hat der erste Kriminologietag zu einer Stärkung dieser Disziplin bereits beigetragen. Eine regelmäßige Wiederholung derartiger Tagungen ist überaus wünschenswert und zu erwarten.

Dr. jur. Michael Jasch ist wissenschaftlicher Mitarbeiter und Lehrbeauftragter am Institut für Kriminalwissenschaften der Johann Wolfgang Goethe-Universität Frankfurt a.M., FB Rechtswissenschaft, sowie Geschäftsführer der Gesellschaft für interdisziplinäre wissenschaftliche Kriminologie (GiwK), Jasch@jur.uni-frankfurt.de

\section{Strafverteidigertag: Feindstrafrecht und Willensfreiheit}

Christine Morgenstern

G roße Fragen auf dem 29. Strafverteidigertag: Brauchen wir ein Parallel-, ein Feindstrafrecht? Oder brauchen wir vielleicht gar keines mehr? Die Veranstalter hatten noch etwas bescheidener, aber nicht weniger grundsätzlich gefragt: »Wen schützt das Strafrecht?« Die 500 Teilnehmer beschäftigten sich vom 4 . - 6. März 2005 in Aachen unter diesem Generalthema mit dem Spannungsverhältnis zwischen Sicherheit und Freiheit. In sechs Arbeitsgruppen ging es um immer längere Strafen und die Sicherungsverwahrung; um gesperrte Zeugen und gesperrte Akten; neue Erkenntnisse zu Willensfreiheit; um das Folterverbot; um europäische Entwicklungen und um die Strafprozessreform.

Konkretisiert wurde das Thema am Freitagabend im engagierten Eröffnungsvortrag von Prof. Dr. Bernhard Haffke, Passau, "Vom Rechtssaat zum Sicherheitsstaat? «Ihm ist eine Erweiterung der klassisch-abwehrrechtlichen Sicht wichtig, die sich auf den Schutz vor Eingriffen des Staates in die Freiheitsrechte des Bürgers konzentriert. Dabei will er sich allerdings nicht dem »Mainstream zeitgenössischen Sicherheitsdenkens « ${ }^{1}$ anschließen, sondern die in der Rechsprechung des Bundesverfassungsgerichts und in der Literatur erarbeiteten Figuren, etwa der "grundrechtlichen Schutzpflichten « oder des "Grundrechts auf Sicherheit«, reflektieren, die ihrerseits auf Wandlungen des Staates hin zu einem »Risikobeherrschungsstaat « reagieren. Mit anderen Worten: Das Strafrecht, obgleich als Reaktion auf begangenes Unrecht konzipiert, »entrinnt nicht der Herrschaft des prospektiven, des präventiven Sicherheitsparadigmas«, es bedarf also einer systemischen Gesamtschau. Er weist in diesem Zusammenhang auf den Wandel des Menschenbildes hin, der nicht erst seit Herbst 2001 - die moderne Gesetzgebung beherrscht: Es ist nun offenbar von Misstrauen geprägt; im Gefahrenvorfeld wird dem Bürger nicht mehr Redlichkeit unterstellt, sondern das Gegenteil ist der Fall, und der Unredliche ist "potenziell gefährlich und muss deshalb beobachtet werden.« Er warnt dann eindringlich davor, das Wuchern von Gefährdungsbekämpfungs- oder »Risikosteuerungsgesetzen«, die alles Heil im Vorrang der Prävention vor der Repression sehen, zu akzeptieren. Er nennt dabei als Beispiele das Terrorund das Geldwäschebekämpfungsgesetz oder das jüngst in Kraft getretene Gesetz zur Förderung der Steuerehrlichkeit; aber auch jene Gesetze, die die Schwelle für Gefahrermittlungseingriffe deutlich herabsetzen, wie zum Beispiel das G10-Gesetz; zeigt auch offen sein Misstrauen gegenüber allem, was das Strafrecht "therapeutisieren " will. Es ist Haffke ein Anliegen, darauf hinzuweisen, dass dieser weitgehenden Sozialkontrolle unter dem Etikett der Prävention nicht dadurch ausgewichen werden kann, dass man solche Aspekte aus dem Strafrecht und Strafprozessrecht sauber heraushält und in ein $\mathrm{Ge}$ fahrenabwehrrecht, d. h. in das Polizeirecht verlagert.

Der Samstag war den sechs Arbeitsgruppen gewidmet, die inhaltlich durch das gut zusammengestellte Materialheft vorbereitet werden konnten. Die Ergebnisse sind inzwischen im Internet unter www.strafverteidigervereinigungen.de abrufbar. Die Arbeitsgruppe 1, die sich mit (lebens-)langen Freiheitsstrafen und der Sicherungsverwahrung beschäftigt hatte, wiederholt alte, aber eben leider nicht obsolete Forderungen unter anderem nach Abschaffung jeglicher Form der Sicherungsverwahrung und verlangt eine Standardisierung und Verbesserung von Prognosegutachten. Die Arbeitsgruppe 2 "Sicherheitsstaat versus Fair Trial/Gesperrte Zeugen und gesperrte Akten« erhebt vor allem die Forderung, dass die staatliche Zurückhaltung von Beweismitten (durch Sperrerklärungen etc.) zumindest in vielen Fällen zu einem Verfahrenshindernis führen muss. Arbeitgruppe 4 nahm sich des Folterverbots bzw. vorgelagerter Maßnahmen an. Hier ist neben der Absage an jegliche Relativierung des Folterverbots die Forderung wichtig, das Zusatzprotokoll zur UN-Antifolterkonvention zügig $\mathrm{zu}$ ratifizieren und umzusetzen. Außerdem müsse ein nationales Gremium zur Kontrolle insbesondere in den Haftanstalten eingesetzt werden. Die Arbeitsgruppe 5 »Europäischer Sicherheitsstaat - Europäischer Polizeistaat? « kritisiert in ihrem Ergebnispapier sehr grundsätzlich die rein sicherheitsorientierte, »bürokratische und utilitaristische« Brüsseler Rechtssetzung in Straf- und Strafverfahrensrecht, die häufig eine Angleichung auf den niedrigsten Rechtsstandard mit sich bringe und konkretisiert das am Beispiel des Europäischen Haftbefehls. Die Arbeitsgruppe befürwortet weitere Vernetzungen und auch die Einrichtung einer "Selbsthilfeorganisation" der Strafverteidiger auf europäischer Ebene, wie etwa einen »Eurodefensor «. Die Arbeitsgruppe 6 schließlich befasste sich mit der Strafprozessreform. Sie bedauert, dass die Vorschläge des Diskussionsentwurfs für eine Reform des Strafverfahrens vom Februar 2004 durch die Justizminister der Länder nicht nur nicht vorangebracht wurden, sondern eher eine "Gegenreform « zu verzeichnen sei. Sie erneuert bzw. erweitert unter anderem die Forderungen nach Ausdehnung der notwendigen Verteidigung für alle Verfahren vor dem Schöffengericht und nach technischen Aufzeichnungen aller Vernehmungen im Ermittlungsverfahren sowie der gesamten Hauptverhandlung.

Die Verfasserin dieses Beitrags nahm an der Arbeitsgruppe $3 \mathrm{mit}$ dem kryptischen Titel »Tun wir, was wir wollen oder wollen wir, was wir tun?« teil. Hier ging es um neue Erkenntnisse der Hirnforschung zur Willensfreiheit und den Diskurs mit Philosophen und - zögernd - auch Juristen, für die diese Frage nach der Existenz oder der bloßen Illusion der Willensfreiheit zentrale Bedeu- 